\title{
Networked international politics
}

\section{Complex interdependence and the diffusion of conflict and peace}

\author{
Han Dorussen, Department of Government, University of Essex \\ Erik A Gartzke, Department of Political Science, University of California, San Diego \\ Oliver Westerwinter, Department of Political Science, University of St. Gallen
}

\begin{abstract}
Network theory and methods are becoming increasingly used to study the causes and consequences of conflict. Network analysis allows researchers to develop a better understanding of the causal dynamics and structural geometry of the complex web of interdependencies at work in the onset, incidence, and diffusion of conflict and peace. This issue features new theoretical and empirical research demonstrating how properly accounting for networked interdependencies has profound implications for our understanding of the processes thought to be responsible for conflict behavior of state and non-state actors. Contributors examine the variation in networks of states and transnational actors to explain outcomes related to international conflict and peace. They highlight how networked interdependencies affect conflict and cooperation in a broad range of areas at the center of international relations scholarship. It is helpful to distinguish between three uses of networks, namely as: 1) as theoretical tools, 2) as measurement tools, and 3) as inferential tools. The introduction discusses each of these uses and shows how the contributions rely on one or several of them. Next, Monte Carlo simulations are used to illustrate one of the strengths of network analysis, namely that it helps researchers to avoid biased inferences when the data generating process underlying the observed data contains extra-dyadic interdependencies.
\end{abstract}

Keywords: conflict and cooperation, network analysis, interdependencies, conflict diffusion, Monte Carlo simulations.

Corresponding author: hdorus@essex.ac.uk. 
At least since the mid-1990s, dyadic data have become increasingly common in large- $n$ quantitative studies (Russett \& Oneal, 1999). In many ways, the research program has been impressive, opening new research agendas, creating new data, and generating innovative theories. In particular, it has been successful in directing research toward international relations, i.e., the interaction between specific states, rather than state behavior more generally. However, research designs specifying the dyad as the unit of analysis are characterized by a basic logical tension. On the one hand, the use of dyads reflects reality in that international politics is interactive, requiring the assessment of relationships in order to provide meaningful insights about behavior. On the other hand, the same interdependencies that call for dyadic research designs also extend beyond pairs of states, questioning the adequacy of focusing exclusively on dyads.

Since the need to consider more complex combinations of relationships is acknowledged in theory, it is not clear why one could ignore this in empirical research. In fact, characterizing relationships in international relations by combining countries in pairs may not always be sufficient or warranted. For example, trade dependence is typically measured as the share of trade with a particular partner. Consequently, trade diversification can simultaneously reduce dependency—which might make conflict more likely—and increase trade openness — reducing the tendency for states to experience disputes. There also appear to be fundamental differences between the actions of a state in joining a multilateral military alliance, such as NATO, and a state that chose to conclude 28 separate bilateral defense agreements. Finally, dyadic data routinely violate the independence of observations assumption; for example, observations for the [USA/Germany] dyad are generally not independent from the [USA/France] dyad, since both dyads contain information for the USA.

Starting already in the 1960s, and originally mainly inspired by work in sociology, network analysis has developed into a powerful tool for analyzing complex interdependencies in the international system (Mitchell, 1969; Barnes, 1969; Hafner-Burton, Kahler \& 
Montgomery, 2009; Maoz, 2011; 2012). In the beginning, however, studies were primarily descriptive mapping linkages among states and identifying clusters of states with dense network connections in the international system (Brams, 1966; Gleditsch, 1967; Skjelsbaek, 1972; Christopherson, 1976). Only recently scholars have begun to move toward a more nuanced analytical approach to studying interdependence and other relationships among states, one that allows the behavior of one actor to be contingent on the actions of multiple others. In this context, researchers started to move beyond the descriptive use of network analysis and begun using it to measure and model complex interdependencies in a broad range of substantive areas. Network theory and methods help illuminate the causes and consequences of conflict because they allow researchers to develop a better understanding of the causal dynamics and structural geometry of the complex web of interdependencies at work in the onset, incidence, and diffusion of conflict and peace. These networked interdependencies have already been shown to have crucial consequences for our understanding of the processes thought to be responsible for the conflict behavior of states (Maoz et al., 2006; Ward, Siverson \& Cao, 2007; Dorussen \& Ward, 2008; 2010; Böhmelt, 2009; Warren, 2010; Cranmer \& Desmarais, 2011; Lupu \& Traag, 2013; Kinne, 2013).

The articles in this special issue build on these initial studies and draw on network theory and methods to open up new areas for research on the causes and consequences of international conflict in an interdependent world. Thus, they highlight how networked interdependencies affect conflict and cooperation in a broad range of areas at the center of international relations scholarship. The contributions examine the role of networked interdependencies across a range of topic areas, such as militarized interstate disputes and war (Gartzke \& Westerwinter, 2016; Minhas, Hoff \& Ward, 2016), international cooperation (Gallop, 2016; Haim, 2016), ethnic conflict (Larson, 2016), alliances (Lupu \& Poast, 2016; Maoz \& Joyce, 2016; Warren, 2016), arms transfers (Kinne, 2016), peacekeeping (Ward \& Dorussen, 2016), human rights protection (Chyzh, 2016), international mediation (Böhmelt, 
2016), and conflict resolution by international non-governmental organizations (Wilson, Davis \& Murdie, 2016). They also offer a number of methodological innovations related to the development and application of network analysis to the study of international relations.

\section{Networks as a theoretical, measurement, and inferential tool}

We define networks structurally as sets of units (nodes) and linkages that indicate the presence or absence of relationships among units (Wasserman \& Faust, 1994). Units can be anything from states, rebel movements, and ethnic groups to national or international governmental or non-governmental organizations and even conflicts as such. Relationships can comprise of trade or ethnic ties, military alliances, bilateral cooperation agreements, United Nations roll-call votes, among many others. We examine the variation in networks of states and transnational actors to explain outcomes related to international conflict and peace. In doing so, the articles distinguish between three uses of networks and each article relies on one or several of these uses: 1) as theoretical tools, 2) as measurement tools, and 3) as inferential tools.

Networks can be used as a theoretical tool to account for extra-dyadic relations among states and other actors in the international system. Most studies of interdependence focus on direct relationships between pairs of actors. Scholars emphasizing the importance of strategic interaction have correctly pointed out that within any pair of states, the actions of one actor are not independent of the behavior of other actors (Signorino, 1999). Once one accepts the idea of the interdependence of intra-dyadic behavior, it would appear unlikely, even peculiar, for interdependence to terminate at the boundary of the dyad (Gartzke \& Gleditsch, 2008; Poast, 2010). Dyads are not isolated bubbles, and what happens in one dyad is likely to be influenced by what happens in the other dyads as well. This, in turn, creates a complex system of interdependencies. In addition to higher-order interdependencies within a single network, interdependencies can also exist between different networks. For example, the pattern of 
connections in the network constituted by joint memberships in intergovernmental organizations (IGOs) that operate in issue areas outside the security domain may shape the decisions of states to form, maintain, or terminate alliances, in addition to exogenous covariates and network interdependencies within the alliance network (Westerwinter, 2016). Similarly, relational patterns in the alliance network may have consequences for who is linked to whom in the trade network (Haim, 2016).

Network analysis is well-suited to analyze and represent such extra-dyadic or more complex types of higher-order interdependence among states and to show how they affect international conflict and peace. By doing so, networks as theoretical tools help scholars to develop new arguments about the causes and consequences of war and peace, and help to expand and refine existing theories, such as bargaining theories of war, informational theories of alliances, and spatial theories of conflict onset. Largely driven by research in economics, strategic, game-theoretic, analyses applied to networks, so-called games on networks, have helped to understand the incentives for actors to make (or break) network ties (Goyal, 2007; Jackson, 2008). In political science, Metternich et al. (2013) have used network games to analyze conflict between the government and potential opposition. Network games have pushed rationalist explanations beyond two-actor games and highlight what information will be shared or held private, as well as how direct and indirect ties affect and constrain strategic decisions. In the special issue, Chyzh (2016), Gallop (2016), Larson (2016), and Ward \& Dorussen (2016), all explain conflict and cooperation as equilibriums in games on networks. Chyzh (2016), for example, examines how states' indirect trade relationships affect their domestic human rights performance and argues that there is an inverse relationship between indirect trade and human rights conditions, providing domestically troubled states with a loophole that allows them to enjoy the benefits of trade without paying the costs of domestic pressure for improved human rights conditions. Gallop (2016) uses the similarity of political institutions (political homophily) and geographical distance to explain the 
willingness of states to develop cooperative linkages. Ward \& Dorussen (2016) also postulate homophily_-in this case of foreign policy preferences as expressed through voting in the UN General Assembly—as a determinant of relative contributions to UN peacekeeping missions. Larson (2016) argues that cross-group ties in the context of inter-ethnic rivalry do not necessarily promote peace. Specifically, if cross-group ties are imposed rather than developed organically, lack of coordination in incomplete networks can lead to protracted retaliation. In all these articles, network models reflect precise theoretical arguments, creating appropriate conditions for tests of innovative hypotheses about the diffusion of conflict and peace in the international system.

Networks can also be used as a tool for measurement. Social network analysis provides a rich methodological toolkit for measuring various properties of individual states, dyads, larger groups, and the state system. Many important concepts in international relations — such as power, clustering, and indirect ties—are difficult to measure when states or dyads are examined in isolation. Measuring these concepts can become more tractable by using a networks approach that takes into account the broader structure of international relations. Maoz \& Joyce (2016) employ agent-based modeling (ABM) to explore how shocks lead to realignment in the alliance network. They use a number of network measures to evaluate 'post-shock' connectivity and consistence at the country, dyad, and systemic levels. The propositions of the ABM are subsequently compared to real-world alliance data for the period 1816-2010. Going beyond considering bilateral alliance ties, Haim (2016) analyzes how the network of international political alliances influences trade flows. He includes the shared number of alliances and shared membership of the same alliance community in standard (dyadic) gravity models of trade, and reports that controlling for indirect alliance ties reduces the importance of bilateral ties. Lupu \& Poast (2016) apply the `k'-adic procedure, outlined by Poast (2010), to model the formation of nonaggression pacts as a multilateral process. For capturing the level of threat and rivalry in the strategic environment, they use the 
analytic density measure of recent rivalry cessation. They find that groups of states with greater densities of recently ended rivalries are significantly more likely to form nonaggression pacts.

Gartzke \& Westerwinter (2016) create a measure that combines network centrality and trade dependence in order to analyze how indirect asymmetric and symmetric trade ties affect conflict among states. The results suggest that indirect trade relationships tend to counteract the effects of bilateral trade relationships, weakening the pacifying effects of symmetry and lowering the conflict-inducing effects of bilateral asymmetry. Wilson, Davis \& Murdie (2016) compile original data on links between international nongovernmental organizations. These data reveal the impact of the network of conflict resolution organizations in lowering belligerence among states and demonstrating the potential relevance of efforts by non-state actors and informal diplomatic channels to defuse or discourage international conflict.

Finally, a third role of network analysis is as a tool for making statistical inferences about the determinants and consequences of conflict and peace among states. While standard statistical models assume that observations are independent, network statistical models, such as exponential random graph models (ERGMs) or stochastic actor-oriented models (SAOMs), relax this assumption (Snijders, 1996; Cranmer \& Desmarais, 2011). Unlike conventional statistical techniques, these models do not only correct for non-independence, but also allow researchers to directly estimate complex endogenous interdependencies and to evaluate how these relationships affect the diffusion of conflict and peace. The inclusion of such endogenous interdependencies in statistical network models should be based on careful theorizing about the causal mechanisms that link extra-dyadic interdependencies and the state behavior of interest. This illustrates how the three uses of network analysis we emphasize-in this case networks as theoretical and inferential tool—are complementary and can strengthen each other. In this special issue, Warren (2016) for example, investigates the linkages between interstate military alliances, international conflict, and domestic democratization using a 
dynamic co-evolutionary actor-oriented network model. Kinne (2016) uses SAOMs to study the effect of weapons cooperation agreements (WCAs) on the global arms trade. He analyzes WCAs as an interdependent network, which co-evolves with the individual-level arms trade activity of states where the SAOM accounts for the mutually endogenous relationship between WCAs and weapons flows. His analysis shows that WCAs have significantly increased weapons flows. Using spatial econometrics, Böhmelt (2016) investigates how international mediation efforts diffuse between conflicts. Focusing on 'substantive' ties between crises in the form of joint crisis characteristics, he is thus able to identify the mechanism by which crises, and all actors therein, are influenced by other crises' mediation. Minhas, Hoff \& Ward (2016) introduce tensor regression that handles the non-independence of observations as well as the dynamic, simultaneous co-evolution of networks. While the use of latent-space tensor models is still largely unexplored in political science, they hold great promise in analyzing the flow of cooperation and conflict in the international system. By further developing and applying statistical network models, the articles in this special issue make innovative methodological contributions to the study of international conflict and peace.

\section{Comparing statistical models: An example using international conflict}

One of the strengths of network analysis is that it helps researchers to avoid biased inferences when the data generating process (DGP) underlying the observed data contains extra-dyadic interdependencies (Cranmer \& Desmarais, 2011). Like omitted variable bias creates problems in multiple regression analysis, extra-dyadic or higher-order network interdependencies can confound relationships between covariates of interest and dyadic outcome variables. In testing hypotheses, the failure to adjust for such confounders can result in an increased Type I error rate; that is, researchers will incorrectly reject the null hypothesis of no relationship more often than if they included the confounders. 
We use Monte Carlo simulations to illustrate how dyadic analyses make it difficult to correctly estimate the effects of a DGP that involves extra-dyadic dependencies. Specifically, we examine type I error rates of inferences about the relationship between the dependent network and covariates that are correlated with elements of the DGP but not part of it. We also illustrate challenges in correctly estimating the coefficient values of variables that are part of the DGP. We use the exponential random graph model (Cranmer \& Desmarais, 2011; Lusher, Koskinen \& Robins, 2013) to generate dyadic data that also contains extra-dyadic interdependence structures. The data include exogenous as well as endogenous extra-dyadic dependencies. This allows us to discuss dyadic models that include network variables in the set of predictors as well as network models that also incorporate endogenous interdependencies within the dependent variable. ${ }^{1}$ For the purposes of illustration and to provide the simulation with a substantive reference point, international conflict provides the context for the data, but the example is hypothetical and not meant to replicate any particular study.

The simulation builds on and extends the analysis of Cranmer \& Desmarais (forthcoming) and is based on the following six steps: First, we create an undirected, binary exogenous network of 192 nodes (suppose these are 192 states that exist in the international system in a given year). We compute the degree for each node in the network and create a dyadic covariate that captures the higher of the two degree scores in a dyad for each pair of nodes in the network. This measures the exogenous network-variable part of the DGP. Substantively, this could for example, refer to degree in the network of military alliances, the network of preferential trade agreements, or the network constituted by joint memberships in intergovernmental organizations.

\footnotetext{
${ }^{1}$ The empirical reality that researchers encounter in their substantive domains of interest is often more complicated than the simple extra-dyadic interdependencies included in our illustration. While ERGMs are capable of incorporating a broad range of extra-dyadic or higher order dependence structures that allow researchers to capture more complicated interdependencies, latent space models as discussed by Minhas, Hoff \& Ward (2016) are an alternative that can help to address some of the weaknesses of ERGMs.
} 
Next, we create a second exogenous covariate that correlates with the higher of the dyadic degree scores variable. For the purpose of illustration, suppose that this variable captures the level of democracy in a dyad.

Third, we create an undirected, binary dependent network. Suppose that this is our network of conflict ties among nations. This network is produced by a DGP that includes an edges effect, the exogenous network variable, a 2-star effect, a 3-star effect, and an effect for clustering. A 2-star effect captures configurations in which a node $i$ is directly connected to two other nodes $j$ and $k$. A 3 -star is a 2-star with an additional node $l$ to which $i$ is directly linked. The clustering effect captures the tendency of nodes in the network to form dense local clusters. Most simply, the tendency toward triangular closure refers to a situation where nodes $i, j$, and $k$ are all directly connected with each other. To operationalize this effect we use the geometrically weighted edgewise shared partner statistic (Hunter, 2007). The vector of parameters that links these five effects to the probability of observing our dependent network is $(-5.5,0.45,0.45,-0.25,-0.25)$. The parameter values are selected to avoid degeneracy.

In step 4, based on the dependent network, we compute another covariate that is correlated to the degree in the dependent network. Specifically, we generate a variable that correlates with the distance between the degree scores within the dyad. In the conflict context, this variable captures how similar two states are in terms of the frequency with which they engage in conflict. These four steps create the data used for the estimations and the comparison of Type I error rates for the variables that are not part of the network DGP but are correlated with its elements. We also compare bias in the coefficient estimates of the exogenous network variable that is part of the network DGP between ERGM and logistic regression models.

Fifth, we estimate three models. The first model is a fully specified ERGM that includes all variables in the network DGP as well as the exogenous covariate that correlates with the exogenous network variable and the item that correlates with degree in the dependent 
network. The second model is a dyadic logistic regression model containing the variable correlated with the exogenous network variable as well as the variable correlated with the dependent network degree. The third model is a dyadic logit comprising the variables of Model 2, but additionally the exogenous network variable. For each model, we save the pvalues of the exogenous variable that is correlated with the exogenous network variable as well as the p-values of the variable that is correlated with the degree in the dependent network. We also store the coefficient estimates for the exogenous network variable for the ERGM and the logistic regression model in which it is included.

Finally, we repeat this five-step procedure 5,000 times to produce a distribution of $\mathrm{p}$ values for each of the two exogenous predictors for each of our three models. We also generate a distribution of 5,000 estimated coefficients for the exogenous network variable for the ERGM and the logistic regression model in which it is included.

We use the stored p-values for the two exogenous variables to calculate Type I error rates for the three models at different levels of statistical significance. Figure 1 presents the Type I error rates generated in our simulations for the variable that is correlated with the exogenous network covariate. The highest Type I error can be observed for the model with the variable correlated with the exogenous network variable while failing to include the exogenous network covariate itself. Across significance levels we almost always find a statistically significant relationship between the exogenous covariate and our dependent network, although this relationship does not actually exist. The Type I error rates of the logit model that includes the exogenous network variable as predictor as well as the fully specified ERGM are considerably lower and very similar. Suppose that the exogenous network variable captures the embeddedness of a dyad in the alliance network and the covariate that is correlated with it, but not part of the network DGP, captures the level of democracy in a dyad. By using a logit specification that does not include the alliance network variable in our conflict model, we would falsely identify a statistically significant relationship between 
democracy and conflict, although the extra-dyadic interdependencies in the alliance network at work in the DGP actually generate the dyadic conflict data. Only by including the alliance network variable (either in a logit or an ERGM model) we are able to discover that democracy is actually not related to conflict behavior, while alliance degrees are.

\section{Type I error rate covariate correlated with exogenous network variable}

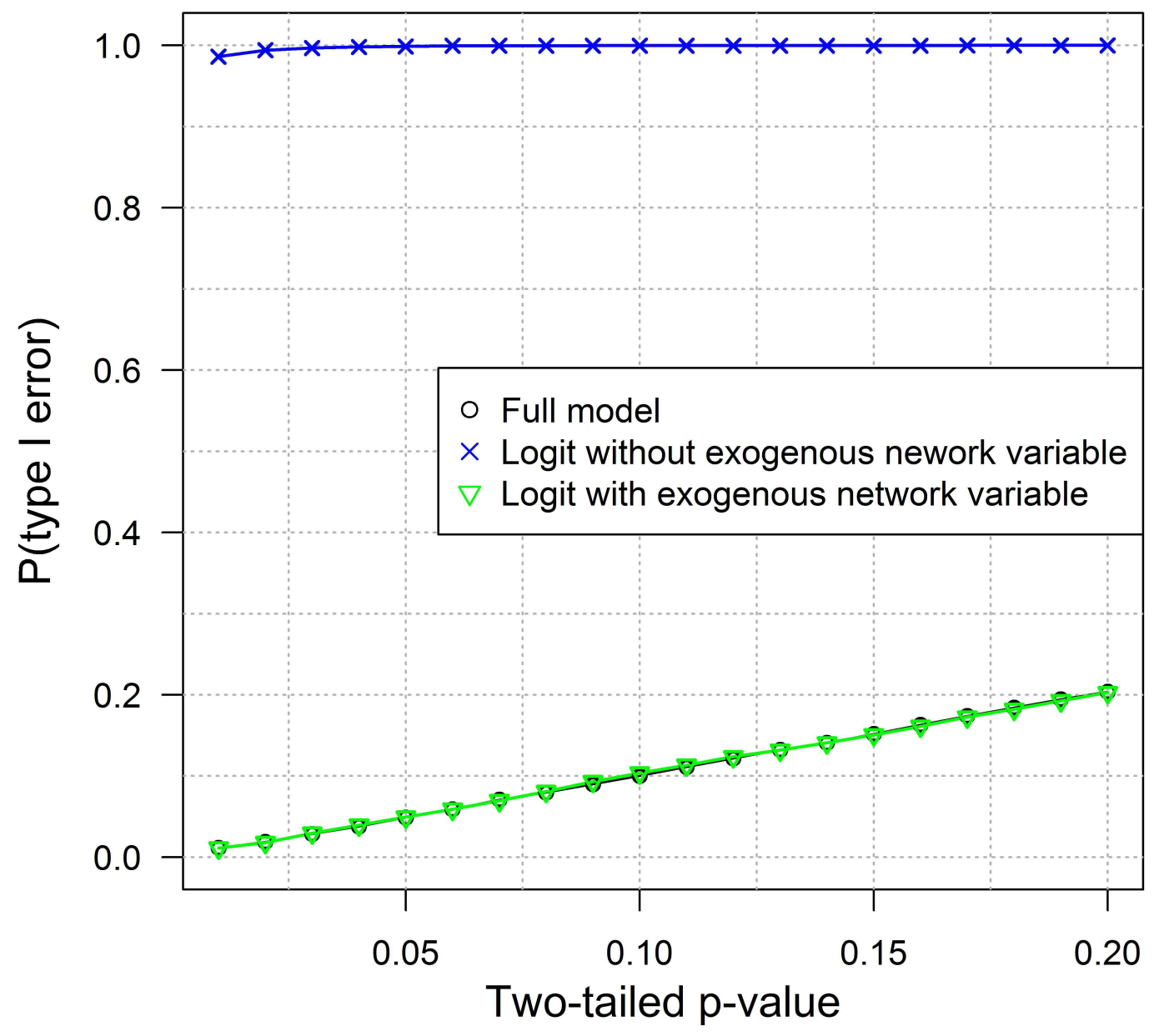

Figure 1. Type I error rates covariate correlated with exogenous network variable. Calculations based on 5,000 repetitions.

Figure 2 shows the results of the Type I error simulations for the covariate that is correlated with the degree distribution in our dependent network, but that is not itself part of the network DGP. Here, we find that the Type I error rates for the fully specified ERGM are 
on average 10 to 45 percent lower than for the logit models with and without the exogenous network variable.

\section{Type I error rate covariate correlated with endogenous network interdependence}

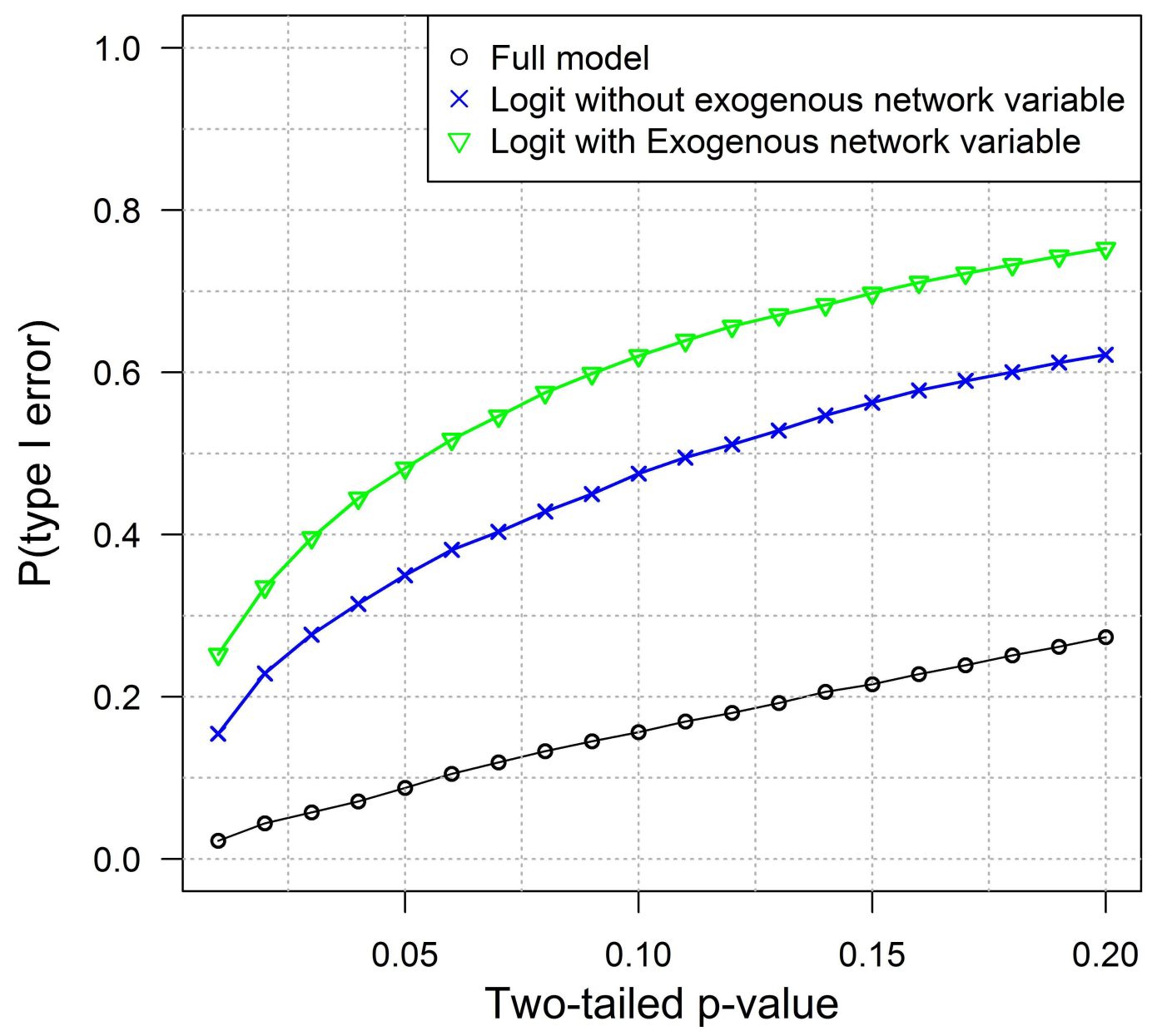

Figure 2. Type I error rates covariate correlated with endogenous network variable. Calculations based on 5,000 repetitions.

The simulations also illustrate how the use of networks as inferential tool can help researchers to avoid bias in their estimates of model coefficients. Focusing on the exogenous network variable, we use bias and root mean squared error to compare the ability of the ERGM and logistic regression to recover the true coefficient that links this variable to our dependent network. Bias is simply the difference between the average value of the coefficient 
estimate in our simulations and the true value of the coefficient. Root mean squared error is calculated as follows: 1) compute the squared difference between each simulation run's coefficient estimate and the true coefficient value; 2) sum up these squared differences and divide by the number of simulation runs; and 3) take the square root of this average. For both measures, the smaller the value, the more accurate the estimation.

Table I. Bias coefficient estimate exogenous network variable (true $\beta=0.45$ )

\begin{tabular}{|l|c|c|}
\hline & $\begin{array}{c}\text { Estimate exogenous network } \\
\text { variable estimated with } \\
\text { ERGM }\end{array}$ & $\begin{array}{c}\text { Estimate exogenous network } \\
\text { variable estimated with logit }\end{array}$ \\
\hline Average $\hat{\beta}$ & 0.464 & 0.142 \\
\hline Bias & 0.014 & -0.308 \\
\hline Root mean squared error & 0.087 & 0.310 \\
\hline
\end{tabular}

Calculations based on 5,000 repetitions.

Column 1 in Table I shows that estimating the effect of the exogenous network variable on the dependent network using the ERGM yields relatively unbiased estimates of this relationship. The average estimated coefficient is very close to the true value of 0.45 and bias and root mean squared error are small. By contrast, logistic regression on average considerably underestimates the effect of the exogenous network variable on the dependent network. This suggests that not including the extra-dyadic interdependencies endogenous to the dependent network produces biased estimates of the true parameter value of the exogenous network variable. In other words, we are likely to err in our estimate of the substantive relationship between the exogenous network variable and our dependent network if our estimation does not include extra-dyadic dependencies within the dependent network.

The simulation illustrates that the omission of extra-dyadic interdependence structures from dyadic models can increase Type I errors through confounding. If seemingly exogenous covariates are correlated with extra-dyadic interdependence structures and included in statistical models without at the same time controlling for these extra-dyadic 
interdependencies, correlation between the extra-dyadic structures and the dependent variable is falsely attributed to the covariate. Consequently, the statistical inferences drawn are likely to be biased, and the failure to include extra-dyadic interdependencies in a dyadic analysis can lead to biased estimates of the coefficient of interest.

By using networks as a tool for measuring extra-dyadic interdependence structures, we can account for these interdependencies in our statistical models and use them as predictors of our dependent variable of interest. In this special issue, Haim (2016), for example, employs measures that capture shared alliances and alliance community membership to predict trade flows. He finds that the inclusion of these extra-dyadic interdependence structures in the alliance network has important consequences for whether we find a statistically significant relationship between dyadic alliance ties and trade. Furthermore, using networks as statistical models allows researchers to consider interdependence structures endogenous to the dependent network into their statistical models. On one hand, as the simulations illustrate, we can avoid faulty statistical inference with respect to the significance of covariates that are correlated with endogenous network interdependencies. We can also considerably reduce the bias in our estimates of coefficients values. On the other hand, network analysis makes it possible for researchers to substantively model the complex interdependence structures that are part of the network DGP. In his contribution to the special issue, Kinne (2016) provides an excellent example for this in the context of WCAs.

\section{Conclusions}

Conventional analyses that highlight the importance of interdependence theoretically often fail to include ties beyond the dyad in their empirical models, creating a gap between theories and empirical tests. Unlike most previous work, the contributions to this special issue take extra-dyadic interdependence explicitly into account and use network theory and methods to incorporate it, both in their theoretical arguments and in the empirical analyses. They show 
that interdependencies of various types play critical roles as determinants for the diffusion of conflict and peace in international affairs, and that analyzing the complex, networked structure of international relations allows us to identify causal mechanisms and explain phenomena related to international conflict and cooperation that may have been overlooked by traditional models.

The studies in this special issue also make important methodological contributions. The tools developed and used by the articles offer new perspectives in the exploration of networked interdependencies in the diffusion of international conflict and peace. They include stochastic actor-oriented models, dynamic models of network co-evolution, latent position network models, networked games, or 'k'-adic analysis. Many of these methods are emerging tools that researchers have only recently begun to adopt in political science and to customize for the study of conflict and peace.

Acknowledgements: We would like to thank Tobias Böhmelt, Olga Chyzh, Yon Lupu, Paul Poast, Henrik Urdal, Hugh Ward, and Michael Ward for helpful comments on previous versions of this article. All mistakes and opinions expressed are those of the authors alone. 


\section{References}

Barnes, John A (1969) Graph theory and social networks: A technical comment on connectedness and connectivity. Sociology 3(2): 215-232.

Böhmelt, Tobias (2016) The importance of conflict characteristics for the diffusion of international mediation. Journal of Peace Research 53(3): XXX-XXX

Böhmelt, Tobias (2009) International mediation and social networks: The importance of indirect ties. International Interactions 35(3): 298-319.

Brams, Steven J (1966) Transaction flows in the international system. American Political Science Review 60(4): 880-898.

Christopherson, Jon (1976) Structural analysis of transaction systems: Vertical fusion of network complexity? Journal of Conflict Resolution 20(4): 637-662.

Chyzh, Olga (2016) Dangerous liaisons: An endogenous model of international trade and human rights. Journal of Peace Research 53(3): XXX-XXX

Cranmer, Skyler J \& Bruce A Desmarais (forthcoming) A critique of dyadic design. International Studies Quarterly.

Cranmer, Skyler J \& Bruce A Desmarais (2011) Inferential network analysis with exponential random graph models. Political Analysis 19(1): 66-86.

Dorussen, Han \& Hugh Ward (2008) Intergovernmental organizations and the Kantian peace. Journal of Conflict Resolution 52(2): 189-212.

Dorussen, Han \& Hugh Ward (2010) Trade networks and the Kantian peace. Journal of Peace Research 47(1): 29-42.

Gallop, Max (2016) Endogenous network and international cooperation. Journal of Peace Research 53(3): XXX-XXX

Gartzke, Erik \& Oliver Westerwinter (2016) The complex structure of commercial peace contrasting trade interdependence, asymmetry and multipolarity. Journal of Peace Research 53(3): XXX-XXX 
Gartzke, Erik A \& Kristian S Gleditsch (2008) The ties that bias. Specifying and operationalizing components of dyadic dependence in international conflict.

Manuscript: University of California, San Diego

(http://citeseerx.ist.psu.edu/viewdoc/download;jsessionid=709FFAF46DEFABB56D2 A8737811135EF?doi=10.1.1.369.3381\&rep=rep1\&type=pdf).

Gleditsch, Nils Petter (1967) Trends in world airline patterns. Journal of Peace Research 4(4): 366-408.

Goyal, Sanjeev (2007) Connections: An Introduction to the Economics of Networks. Princeton NJ: Princeton University Press.

Hafner-Burton, Emilie M; Miles Kahler \& Alexander H Montgomery (2009) Network analysis for international relations. International Organization 63(3): 559-592.

Haim, Dotan (2016) Alliance networks and trade: The effect of indirect political alliances on bilateral trade flows. Journal of Peace Research 53(3): XXX-XXX

Hunter, David R (2007) Curved exponential family models for social networks. Social Networks 29(2): 216-230.

Jackson, Matthew O (2008) Social and Economic Networks. Princeton: Princeton University Press.

Kinne, Brandon (2016) Agreeing to arm: Bilateral weapons agreements and the global arms trade. Journal of Peace Research 53(3): XXX-XXX

Kinne, Brandon J (2013) Network dynamics and the evolution of international cooperation. American Political Science Review 107(4): 766-785.

Larson, Jennifer M (2016) Interethnic conflict and the dangers of cross-group ties. Journal of Peace Research 53(3): XXX-XXX

Lupu, Yonatan \& Paul Poast (2016) Teams of former rivals: A multilateral theory of nonaggression pacts. Journal of Peace Research 53(3): XXX-XXX 
Lupu, Yonatan \& Vincent A Traag (2013) Trading communities, the networked structure of international relations, and the Kantian peace. Journal of Conflict Resolution 57(6): 1011-1042.

Lusher, Dean; Johan Koskinen \& Garry Robins (2013) Exponential Random Graph Models for Social Networks. Cambridge: Cambridge University Press.

Maoz, Zeev (2012) How network analysis can inform the study of international relations. Conflict Management and Peace Science 29(3): 247-256.

Maoz, Zeev (2011) Networks of Nations. The Evolution, Structure, and Impact of International Networks, 1816-2001. Cambridge: Cambridge University Press.

Maoz, Zeev \& Kyle Joyce (2016) The Effects of shocks on international networks: Changes in the attributes of states and the structure of international alliance networks. Journal of Peace Research 53(3): XXX-XXX

Maoz, Zeev; Ranan D Kuperman, Lesley Terris \& Ilan Talmud (2006) Structural equivalence and international conflict. Journal of Conflict Resolution 50(5): 664-689.

Metternich, Nils; Cassy Dorff, Max Gallup, Simon Weschle \& Michael D Ward (2013) Antigovernment networks in civil conflicts. How network structures affect antigovernment behavior. American Journal of Political Science 57(4): 892-911.

Minhas, Shahryar; Peter D Hoff \& Michael D Ward (2016) Relax, tensors are here: Forecasts of dyadic conflict networks. Journal of Peace Research 53(3): XXX-XXX

Mitchell, Clyde J (1969) The concept and use of social networks. In: Clyde J Mitchell (ed.) Social Networks in Urban Situations. Manchester: Manchester University Press, 1-50.

Russett, Bruce \& John R Oneal (2001) Triangulating peace: Democracy, interdependence, and international organizations. New York: Norton.

Signorino, Curtis S (1999) Strategic interaction and the statistical analysis of international conflict. American Political Science Review 93(2): 279-297. 
Skjelsbæk, Kjell (1972) Peace and the structure of the international organization network. Journal of Peace Research 99(4): 315-330.

Snijders, Tom A B (1996) Stochastic actor-oriented models for network change. Journal of Mathematical Sociology 21(1-2): 149-172.

Ward, Hugh \& Han Dorussen (2016) Standing alongside your friends: Jointly produced private benefits, network centrality, and the provision of troops for UN peacekeeping operations. Journal of Peace Research 53(3): XXX-XXX

Warren, T Camber (2016) Modeling the co-evolution of international and domestic institutions: Alliances, democracy, and the complex path to peace. Journal of Peace Research 53(3): XXX-XXX

Warren, T Camber (2010) The geometry of security: Modeling interstate alliances as evolving networks. Journal of Peace Research 47(6): 697-709.

Ward, Michael D; Randolph M Siverson \& Xun Cao (2007) Disputes, democracies, and dependencies: A reexamination of the Kantian peace. American Journal of Political Science 51(3): 583-601.

Wasserman, Stanley \& Katherine Faust (1994) Social Network Analysis: Methods and Applications. Cambridge: Cambridge University Press.

Westerwinter, Oliver (2016) Multiplex choices: Intra- and inter-network dependencies and the evolution of military alliances. Manuscript: University of St. Gallen.

Wilson, Maya; David R Davis \& Amanda Murdie (2016) The view from the bottom: Networks of conflict resolution organizations and international peace. Journal of Peace Research 53(3): XXX-XXX 
HAN DORUSSEN, b. 1962, PhD in Political Science (University of Texas, Austin 1996); Professor, Department of Government, University of Essex (2009- ); current main interests: trade and conflict, peacekeeping, and peacebuilding.

ERIK GARTZKE, b. 1965, PhD (University of Iowa, 1997); Professor (2014-), Associate Professor (2007-2014), University of California, San Diego; has held faculty positions at the University of Essex, Columbia University and the Pennsylvania State University. Research interests primarily involve the causes of war and peace.

OLIVER WESTERWINTER, b. 1983, PhD in Political Science (European University Institute, 2014); Assistant Professor, Department of Political Science, University St. Gallen (2016-); post-doctoral research fellow (2013-2016), University of St. Gallen; visiting fellow (2011), University of California, San Diego, School of International Relations and Pacific Studies; current main research interests: international conflict, alliances, bargaining models, network theory, informal institutions, and political methodology. 